Methods: The prospective study included 109 women aged 38-75 y.o., of I-III Kellgren J. - Lawrence J. stage of knee OA (ACR), who signed an informed consent. The average age was $59.3 \pm 8.7$ y.o. (from 38 to 74 ), the average duration of the OA was 7 (4-12) years. The average values of the body mass index (BMI) corresponded to obesity $\left(30.9 \pm 5.4 \mathrm{~kg} / \mathrm{m}^{2}\right)$, waist circumference $(\mathrm{WC})-94.4 \pm$ $11.7 \mathrm{~cm}$. An individual card was filled in for each patient, including anthropometric parameters, anamnesis and clinical examination data, assessment of knee joint pain according to VAS, WOMAC, KOOS and DN4 indices, and concomitant diseases. All patients underwent standard X-ray of the knee joints, laboratory examination.

Results: AH was diagnosed in $69.7 \%$ patients with OA. Patients were divided into 2 groups, according to the presence or absence of $\mathrm{AH}$ (Table 1). Patients with $\mathrm{AH}$ were older, had a higher $\mathrm{BMI}, \mathrm{WC}$, a longer duration of menopause and significantly earlier its onset $(p<0.05)$. Patients with $O A$ and $A H$ had a more severe course of OA: higher values of pain in VAS, total WOMAC and all its components, DN4, worse indicators of total KOOS $(p<0.05)$. X-ray examination showed a tendency to a more significant narrowing of the medial space of the knee joints $(p=0.07)$. Laboratory examination showed higher values of CRP, ESR, IL-6, and leptin $(p<0.05)$.

Table 1. Comparative characteristics of $\mathrm{OA}$ patients with and without $\mathrm{AH}$

\begin{tabular}{lccc}
\hline Parameters & $\begin{array}{c}\text { Patients with AH } \\
(\mathrm{n}=76)\end{array}$ & $\begin{array}{c}\text { Patients without AH } \\
(\mathrm{n}=33)\end{array}$ & $\mathrm{p}$ \\
\hline Age, y.o. & $61(57-68)$ & $55.5(49-58)$ & $<0.01$ \\
WC, cm & $92(90-105)$ & $86.5(84-90)$ & $<0.01$ \\
Age of menopause, y.o. & $50(47-52)$ & $55.5(49-58)$ & 0.02 \\
Duration of menopause, years & $14(7.5-19)$ & $7(4-8)$ & $<0.01$ \\
Duration of OA, years & $10(5-15)$ & $4(1-6)$ & 0.001 \\
VAS pain score, mm & $49(40-57)$ & $42(24-50)$ & 0.02 \\
WOMAC pain, mm & $189.5(140-250)$ & $140(108-162)$ & 0.001 \\
WOMAC stiffness, mm & $77.5(42-100)$ & $56(33.5-71.5)$ & 0.01 \\
WOMAC functional impairment (FI), mm & $651(547-902)$ & $546.5(320-663.5)$ & 0.002 \\
Total WOMAC, mm & $899(728-1280)$ & $734(526.5-882)$ & 0.001 \\
KOOS, points & $0.47(0.36-0.57)$ & $0.6(0.53-0.75)$ & $<0.01$ \\
DN4, points & $2(1-3)$ & $1(0-2)$ & 0.01 \\
Overall health status, mm & $45(35-55)$ & $36.5(28.5-48.5)$ & 0.02 \\
The size of the medial space of joint accord- & $2.45(1.35-4.35)$ & $3.6(2.8-4.3)$ & 0.07 \\
$\quad$ ing to X-ray, mm & & & \\
CRP, mg/l & $2.38(1.47-4.85)$ & $1.21(0.69-2.53)$ & $<0.01$ \\
Leptin, $\mathrm{ng} / \mathrm{ml}$ & $37.4(26.5-53.3)$ & $23.6(15.1-40.2)$ & 0.01 \\
IL-6, pg/ml & $0.7(0.4-1.2)$ & $0.45(0.3-0.7)$ & 0.03 \\
ESR, mm/h & $14(7-18)$ & $7(6-12)$ & 0.02 \\
\hline
\end{tabular}

We founded positive $(\mathrm{p}<0.05)$ associations between $\mathrm{AH}$ and a more severe, prolonged course of $O A(r=-0.39, p<0.01)$ in the the Spearman rank-order correlation coefficient analysis. Thus, patients with $\mathrm{AH}$ had higher values of VAS pain $(r=0.31, p<0.01)$, total WOMAC $(r=0.31, p<0.01)$ and all its components (pain $(r=0.33, p<0.01), F I(r=0.3, p<0.01)$ and stiffness $(r=0.24, p<0.01)$, DN4 $(r=0.24$, $p=0.01)$, worse indicators of total KOOS $(r=-0.42, p<0.01)$ and overall health status $(r=0.23, p=0.02)$, more often detected more advanced stage of $O A(r=0.24$, $p=0.03)$ and synovitis $(r=0.23, p=0.01)$. In addition, positive relationships were found with CRP ( $r=0.31, p<0.01)$, IL - $6(r=0.3, p=0.03)$, ESR $(r=0.3, p=0.02)$. Positive relationships were confirmed between $\mathrm{AH}$ and age $(r=0.39, p<0.01)$, menopause duration $(r=0.39, p<0.01)$, WC $(r=0.37, p<0.01)$, leptin $(r=0.35$, $p=0.01)$, the presence of hypertriglyceridemia $(r=0.35, p=0.01)$ and cardiovascular risks according to SCORE $(r=0.26, p=0.02)$, considering traditional risk factors for cardiovascular diseases (CVD).

Conclusion: Thus, we found that $\mathrm{AH}$ in patients with knee $\mathrm{OA}$ is affected by a variety of variables, both related to traditional CVD factors and to OA itself, and the correlations found are approximately equal in strength. The results obtained require further study, and it is possible that preventive measures aimed at reducing the traditional risk factors of diseases of the circulatory system, or correcting existing CVD, will contribute to a more favorable course of OA.

Disclosure of Interests: None declared

DOI: 10.1136/annrheumdis-2021-eular.3859

\section{POS0131 10-YEAR PROBABILITY OF A MAJOR OSTEOPOROTIC FRACTURES IN WOMEN WITH OSTEOARTHRITIS OF THE KNEE JOINT}

E. Usova $^{1}$, O. Malyshenko ${ }^{1}$, M. Letaeva ${ }^{1}$, J. Averkieva ${ }^{1}$, M. Koroleva ${ }^{1}$, T. Raskina'. 'State Educational Institution of Higher Education "Kemerovo State Medical University", Kemerovo, Russian Federation, Department of Internal Diseases, Kemerovo, Russian Federation

Background: The relationship between osteoporosis and osteoarthritis (OA) is complex and contradictory. Some studies suggest a protective effect of $O A$ in osteoporosis [1-2]. However, other studies show that increased bone mineral density (BMD) in OA not only does not reduce the risk of fractures, but can also increase it [3-4].

Objectives: To assess the 10-year probability of osteoporotic fractures using the FRAX calculator in women with OA of the knee joint.

Methods: The study included 22 women (average age $63.7 \pm 1.01$ years) diagnosed with ACP of the knee joint according to the ACR criteria (1991). The Control Group included 24 conditionally healthy women without OA knee joint, with an average age of $63.6 \pm 1.37$ years.

The BMD $(\mathrm{g} / \mathrm{cm} 2)$ and the T-criterion (standard deviation, SD) of the neck of the femur and lumbar spine (LI-LIV) were evaluated by the method of two-power X-ray absorption (DXA) (apparatus «Lunar Prodigy Primo», USA). 10-year probability of major osteoporotic fractures (clinically significant fracture of the spine, distal fracture of the forearm, fracture of the proximal femur, or fracture of the shoulder) and fracture of the proximal thigh with the FRAX calculator (version 3.5 for Russian population)

Results: An osteopenic syndrome in the cohort under investigation was found in $42(91.3 \%)$ patients, of whom osteopenia in $24(52.2 \%)$ women and osteoporosis in $18(39.1 \%)$. A normal BMD is registered in $4(8.7 \%)$ patients.

In the group of patients with knee joint OA, only $2(9.1 \%)$ of women had a normal BMD, $11(50.0 \%)$ of osteoporosis, and $9(40.9 \%)$. Osteopenic syndrome is generally found in $20(90,9 \%)$ patients.

In the control group, osteopenic syndrome has been diagnosed in $22(91,7 \%)$ of whom: osteopenia in $13(54.2 \%)$, osteoporosis in 9 (37.5\%) patients. Two (8.3\%) women had a normal BMD. There were no statistically significant differences in the structure of the osteopenic syndrome among the studied groups $(p=0.961)$. An analysis of the 10-year probability of major osteoporotic fractures found that women with OA knee joint had the above probability of $12.3 \pm 0.91$, and in the control group $14.2 \pm 1.06(\mathrm{p}=0.085)$.

The 10-year probability of fracture of the proximal femur in women with OA was statistically less significant than in the control group: $1.55(0.70 ; 1.98)$ and 2.10 $(1.20 ; 2.95),(p=0.031)$, respectively.

Conclusion: The total incidence of the osteopenic syndrome in the cohort under investigation was $91.3 \%$ (90.9\% in women with OA, $91.7 \%$ in the control group) The frequency of registration of osteopenia and osteoporosis in women with $\mathrm{OA}$ did not differ statistically significantly from the control group. The probability of major osteoporotic fractures within 10 years was comparable in these groups. The probability of a proximal femur fracture in women with OA was statistically significant, but not clinically significant, compared to the control group.

REFERENCES:

[1] Yamamoto $Y$, Turkiewicz A, Wingstrand $H$, et al. Fragility Fractures in Patients with Rheumatoid Arthritis and Osteoarthritis Compared with the General Population. J Rheumatol. 2015 Nov;42(11):2055-8.

[2] Vala $\mathrm{CH}$, Kärrholm J, Kanis JA, et al. Risk for hip fracture before and after total knee replacement in Sweden. Osteoporos Int. 2020 May;31(5):887-895

[3] Kim BY, Kim HA, Jung JY, et al. Clinical Impact of the Fracture Risk Assessment Tool on the Treatment Decision for Osteoporosis in Patients with Knee Osteoarthritis: A Multicenter Comparative Study of the Fracture Risk Assess ment Tool and World Health Organization Criteria. J Clin Med. 2019 Jun 26;8(7):918.

[4] Soh SE, Barker AL, Morello RT, et al. Applying the International Classification of Functioning, Disability and Health framework to determine the predictors of falls and fractures in people with osteoarthritis or at high risk of developing osteoarthritis: data from the Osteoarthritis Initiative. BMC Musculoskelet Disord. 2020 Feb 29;21(1):138.

Disclosure of Interests: None declared

DOI: 10.1136/annrheumdis-2021-eular.3446

\section{Crystal arthritis}

\section{POS0132 IS THE INTERCRITICAL GOUT REALLY ASYMPTOMATIC? THE INFLAMMATORY ROLE OF THE SILENT URATE CRYSTAL DEPOSITION}

C. Diaz-Torne ${ }^{1}$, M. A. Ortiz ${ }^{2}$, S. Jeria Navarro ${ }^{1}$, A. Garcia-Gullien ${ }^{1}$, L. Sainz ${ }^{1}$, H. Corominas ${ }^{1}$, S. Vidal ${ }^{2} .{ }^{1}$ Hospital de la Santa Creu i Sant Pau, Rheumatology, Barcelona, Spain; ${ }^{2}$ Institut de Recerca Hospital de la Santa Creu i Sant Pau, Immunology, Barcelona, Spain

Background: Gout is the most prevalent inflammatory arthritis. Gout is chronic inflammatory deposition disease related to an increase of cardiovascular (CV) events and mortality. Subclinical chronic inflammation has been demonstrated in this patients but not its relation with the monosodium urate (MSU) crystal deposit size and the number of CV risk factors. 